\title{
Axonal polineuropathy associated with Sars-CoV 2 infection: a case report
}

\author{
Caterina Tramonti, Elena Vatteroni, Francesco Iacopini, Valentina Carli, Martina \\ Iardella
}

IRCCS Fondazione Don Carlo Gnocchi Onlus, Firenze, Italy

This article is distributed under the terms of the Creative Commons Attribution Noncommercial License (CC BY-NC 4.0) which permits any noncommercial use, distribution, and reproduction in any medium, provided the original author(s) and source are credited.

\begin{abstract}
World healthcare systems are dealing with the pandemic of the severe Coronavirus disease 19 (Covid-19). Covid-19 neurological manifestations affecting both central and peripheral nervous systems have been reported. We describe the case of 44-year-old patient, who was admitted to our Rehabilitation Unit after discharge from intensive care unit for severe Covid-19 related respiratory failure. Neurophysiological investigation revealed an axonal polineuropathy with impairment both of sensory and motor component. He underwent a comprehensive rehabilitation treatment, including breathing exercises and motor rehabilitation. Despite rapid and effective diagnosis and customized progressive rehabilitation treatment, significant improvement in sensory-motor abnormalities has begun but distal complete recovery was not achieved. Indeed, complete indipendence during walking has been partially reached: patient constantly needs two sticks and wears two ankle-foot orthosis during ambulatory function. Nowadays, exploring the neurologic manifestations of Covid-19 may represent a step towards better understanding the virus, preventing longterm sequela and rehabilitation concerns.
\end{abstract}

Key Words: Covid-19; neurological rehabilitation; exercise therapy; peripheral nervous system. Eur J Transl Myol 31 (3): 9900, 2021 doi: 10.4081/ejtm.2021.9900

\begin{abstract}
Nowadays, world healthcare systems are dealing with the pandemic of the severe acute respiratory coronavirus 2 (SARS-CoV-2) and its associated disease, named Coronavirus disease 19 (Covid-19). ${ }^{1}$ Covid-19 infection mostly affects the respiratory system, with clinical presentation ranging from mild flu-like symptoms to severe acute respiratory infection. Anyway, extrarespiratory multisystemic involvement has also been reported, ${ }^{2}$ with neurological complications affecting both central and peripheral nervous systems. ${ }^{3}$ To the best of current knowledge, observational investigations reported peripheral nervous system involvement in up to $8.9 \%$ of people. ${ }^{4}$ Although mechanisms of Covid-19related neuropathy are yet not fully understood, the possibility of SARS-CoV-2 as a new neuropathogen has been postulated. ${ }^{5}$ Therefore, exploring the neurologic manifestations of Covid-19 may represent a step towards better understanding the virus, preventing longterm sequela and rehabilitation concerns.
\end{abstract}

\section{Materials and Methods}

Case Report

We describe the case of a 44-year-old male patient, who underwent Covid-19 infection. He reported the onset of mild-flu like syndrome (fever, chills, headache, myalgia, cough and fatigue) from March 30, 2020. His nasopharyngeal specimen was tested for reverse transcription-polymerase chain reaction (RT-PCR) and its result was positive. Then, he was referred to emergency ward for serious respiratory failure needing oxygen supply with cPAP and "casco" (continuos positive airway pressure). Chest computed tomography (CT) scan showed multiple bilateral ground glass opacities and consolidations, typical of Covid-19 pneumonia (Figure 1). He denied other symptoms, tobacco and drugs use or abuse. Significant past medical history comprehended diagnosis of diabetes mellitus type 2 , obesity and a mild cognitive impairment in a psychotic disorder. Then, the patient was rapidly transferred to an intensive-care unit for the severe deterioration of his respiratory condition. Intensive-care clinical course was complicated by:

- Acute renal failure, without dialysis treatment;

- Bacteric pulmonary sovrainfection, with necessity of specific antibiotic treatment;

- Complete atelectasia of left lung. Patient underwent a tracheostomy to support adequate respiratory function;

- Systemic sepsis and urinary tract infection, treated with specific antibiotic therapy;

- High blood pressure levels, with the need to start an antihypertensive therapy. 
a.

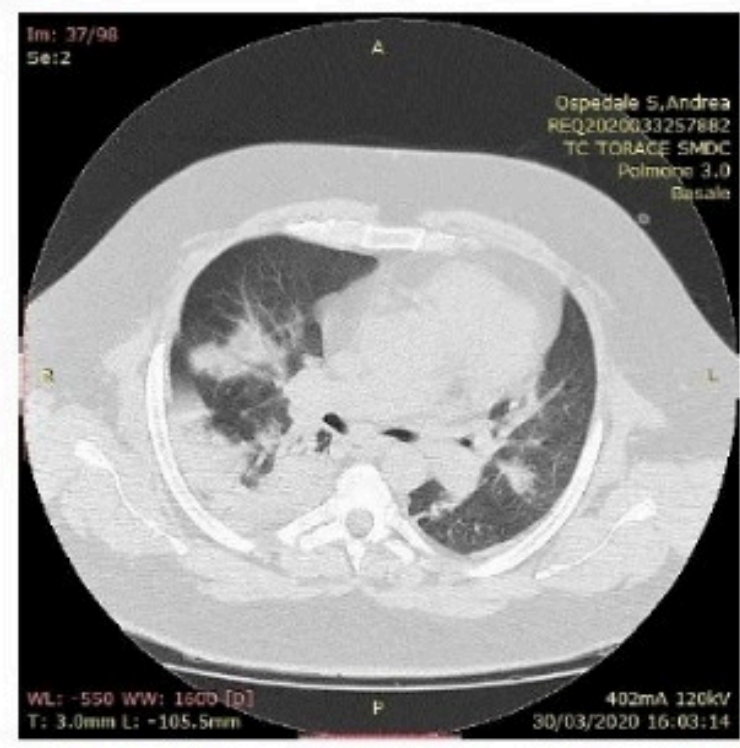

b.

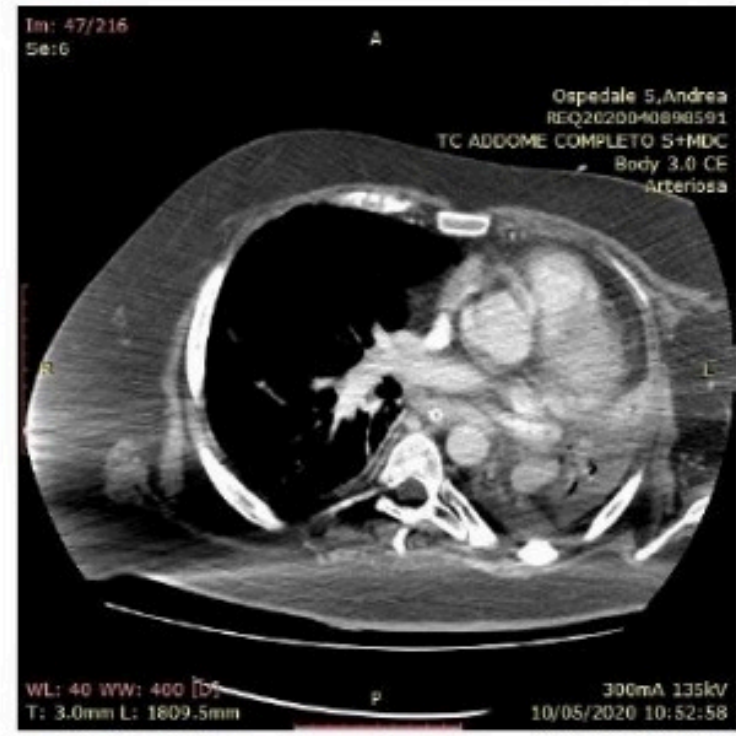

Fig 1. Chest CT scan, at the beginning (a) and after two months (b) of recovery period.

Once stabilization of clinial conditions, the patient was transferrred to our Rehabilitation ward. Neurologic examination showed normal consciousness by Glasgow Coma Scale (GCS=15) and no cranial nerves abnormality. The upper and lower limbs strength was evaluated by means of manual muscle testing using the Medical Research Council scale (MRC): ${ }^{6}$ he was scored 3 at shoulder abduction and handgrip, and 4 at elbow flexion, hip and knee extension; he was scored 1 at ankle dorsiflexion, bilaterally. Clinical examination also revealed absent deep tendon reflexes in upper and lower limbs and normal plantar response, symmetrically. No sensitivity alteration was assessed. Evaluation of respiratory gas exchanges revealed a good balance, with no need for oxygen supply. Neurophysiological investigation revealed an axonal polineuropathy with impairment both of sensory and motor component (Figure 2): absence of both sural nerve sensory action

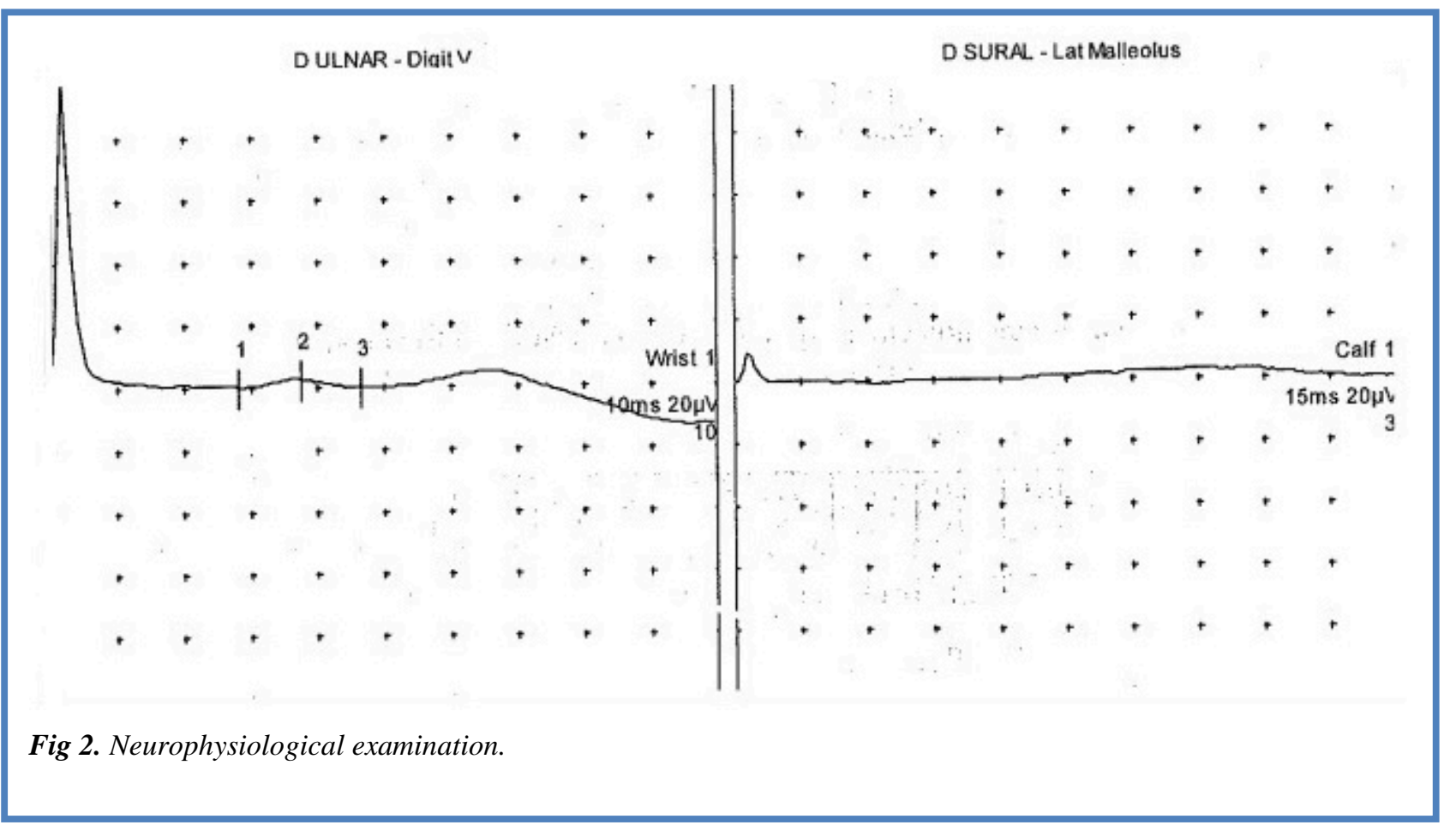


3.a

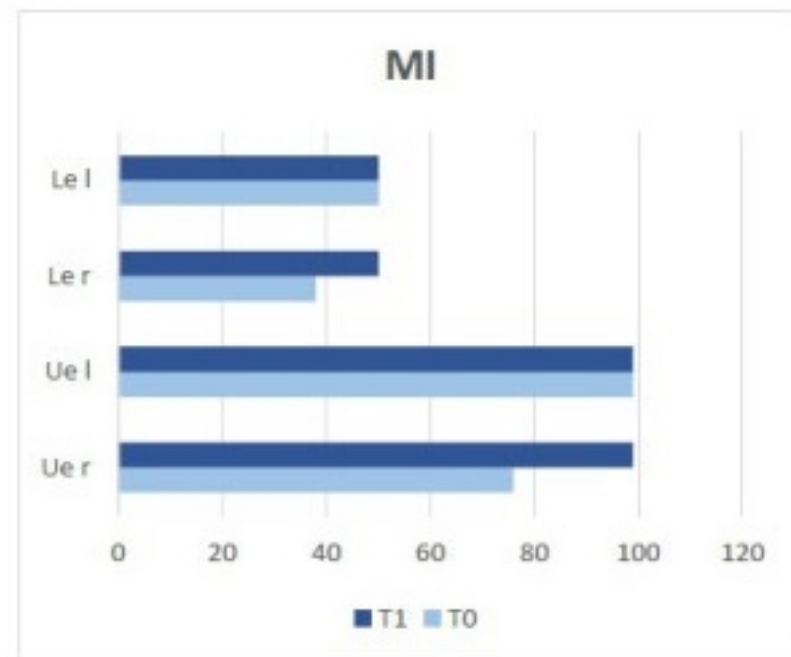

3.b

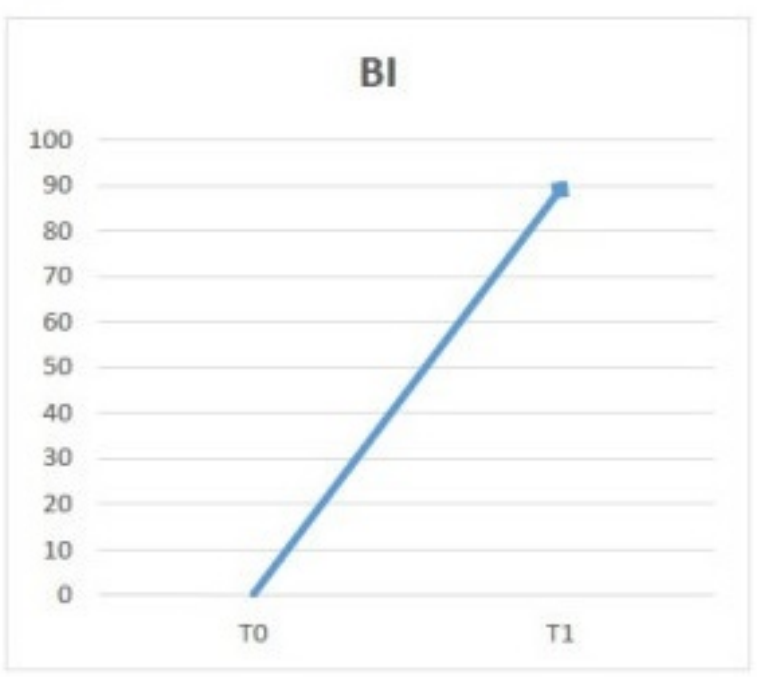

Abbreviations: MI: Motricity Index; BI: Barthel Index; Ue: upper extremities; Le: lower extremities; r: right; l: left.

Fig 3. Functional scales.

potential (SAP) and the common peroneal nerve compound muscle action potential (CMAP), decreased tibial nerve velocity and severely decreased CMAP amplitude for the same nerve on electroneurography, and decreased SAP amplitude for ulnar nerve. Patient's functional status and disability were assessed by means of Motricity Index, ${ }^{7}$ Timed Up and Go Test (TUG) ${ }^{8}$ and Barthel Index. ${ }^{9}$

\section{Training Protocol}

The patient received a comprehensive rehabilitation treatment, including respiratory and motor rehabilitation. The training program was organized in 5 sessions per week, each session lasting about 60 minutes. The overall duration of rehabilitation program was about 9 months, as long as hospitalization period. The training program was conceived with a progressive evolution and intensity, where the progression is specifically tailored on patients' competencies and motor functions improvement. At the beginning, physiotherapy was manly focused on daily standardized passive or active motion session of the upper and lower limbs and active postural changes. Breathing exercises for respiratory muscle were focused on chest expansion, controlled breathing and diaphragmatic re-education. According to patients' functional improvements, the programme included coordination exercises for trunk control, recovery of standing position, reconditioning of walking and functional activities. Finally, an overground ambulatory training with the prescription of orthosis was administered in order to balance distal neurological deficits.

\section{Results}

Considering functional scales, we revealed an improvement of mobility as shown by the possibility to perform TUG test at the end of recovery period, (TUG at $\mathrm{T} 1=20.29$ s). Moreover, we showed a partial improvement of patient's motor abilities as assessed by Motricity Index. In particular, we found a complete recovery for upper extremities abilities on the right side (T0: 76 vs T1:100); while, lower extremities function remained quite impaired at the end of rehabilitation period, with a stationary situation for the left side (T0: 50 vs $\mathrm{T} 1: 50$ ) and a mild improvement for the right side (T0: 38 vs T1:50), Figure 3a. As regard lower extremities muscle, we found no improvement in distal district, as suggested by complete absence of ankle dorsiflexors activity. Finally, overall functional abilities were improved after rehabilitation training, as evidenced by Barthel Index score (T0: 0 vs T1: 89), Figure 3b. In particular, our patient gained more advantage in self-care activities, such as feeding and bathing, and in mobility, such as during postural transfers and ambulatory function.

\section{Discussion}

Nowadays, extrarespiratory multi-systemic involvement of SARS-CoV-2 has been reported, with neurological manifestations appearing in the acute phase and worsening functional outcomes after training period. In this context, we describe the case of young male who underwent Covid-19 infection, resulting in prevalent neurological impairment: axonal polineuropathy with impairment both of sensory and motor component. Despite rapid and effective diagnosis and customized 
long-lasting progressive rehabilitation treatment, significant improvement in functional disabilities has begun but distal complete recovery was not achieved. Indeed, complete indipendence during walking has been partially reached: patient constantly needs two sticks and wears two ankle-foot orthosis during ambulatory function. According to the actual hypothesis of a possible neurotropism of SARS-CoV-2, the mechanism of Covid19 related neuropathy are yet to be fully evaluated. ${ }^{4}$ As known, infectious peripheral neuropathy may occur secondary to other viruses such as hepatitis C, human immunodeficiency virus (HIV) and varicella zoster. ${ }^{6}$ Anyway, acute polyneuropathies has been documented in patients infected with SARS-CoV and MERS-CoV. ${ }^{10,11}$ Therefore, acute autoimmune polyneuropathy triggered by coronavirus infection has been postulated, according to its neurotrophic, neuroinvasive, and neuroinflammatory features. ${ }^{12}$ As found in case report publications, most of patients with polyneuropathy were observed in a critically ill context, as well as our patient. Indeed, prolonged hospitalization can itself lead to peripheral nerve pathology due to critical illness polyneuropathy (CIP) or secondary to prolonged patient positioning. Moreover, prolonged neuromuscular blockage, vitamin deficiencies and electrolyte disturbances, and drug related neuromuscular disorders have to be included in the differential diagnosis list. ${ }^{6}$

A recent case report, including 4 patients with acute neuromuscular disorders and SARS infection, described the onset of weakness, sensorimotor peripheral nerve symptoms and decreased deep tendon reflexes within 2125 days after the onset of SARS. Neurophysiological studies indicated temporarily reduced CMAP amplitudes, with no evidence of reduced nerve conduction velocity, prolonged distal motor latency, conduction block or temporal dispersion. Electromyography showed acute denervation with increased polyphasia. As far as these patients received intensive care for multiple organ failure, it could be argued that CIP likely played a significant role. ${ }^{12}$ A single case report showed a 28-year-old male with a MERS$\mathrm{CoV}$ severe respiratory infection that gradually improved. He was found to have weakness in both legs and an inability to walk, and nerve conduction study showed axonal polyneuropathy. A CIP was assumed as the final diagnosis. ${ }^{10}$ Another report studying acute polyneuropathy in $\mathrm{CoV}$ included 4 patients with Guillain-Barré syndrome (GBS) attributed to the MERS$\mathrm{CoV}$ infection. Two of these patients were diagnosed with acute sensory neuropathy in the context of treatment with ribavirin and lopinavir/ ritonavir. ${ }^{12}$ Similarly, a 3 patient case report showed 2 women experiencing motorpredominant peripheral nerve disorders and another woman experiencing neuropathy and myopathy 3 weeks after the onset of SARS-CoV-2. Clinical and electrophysiologic improvement was evident during follow-up examinations, with a good prognosis. ${ }^{11}$ Based on the current literature, mechanisms of peripheral nerve injury in Covid-19 patients may be multifactorial: complications of systemic disease, direct neuro-invasion and autoimmune response may have promoted peripheral nerve injury even in our subject. However, developing axonal polyneuropathies in the context of a viral infection suggests that the virus can cause a neural inflammatory reaction through immune mimicry or present as part of an inflammatory response syndrome. Those mechanism of SARS-CoV-2 related neuropathy needs to be clarified in order to promote a correct management and prevent patient morbidity.

In conclusion, since patients recovery from COVID-19 infection include a variety of pulmonary, neurological and cognitive complications, management of peripheral nerve injury in COVID-19 survivors should be included in future COVID-19-specific rehabilitation programs in order to prevent long-term sequelae.

\section{List of acronyms}

CIP - critical illness polyneuropathy

CMAP - compound muscle action potential

Covid-19 - Coronavirus disease 19

cPAP - continuos positive airway pressure

CT - computed tomography

GBS - Guillain-Barré syndrome

GCS - Glasgow Coma Scale

HIV - human immunodeficiency virus

MERS-CoV - Middle East Respiratory SyndromeCoronavirus

MRC - Medical Research Council

RT-PCR - reverse transcription-polymerase chain reaction

SAP - sensory action potential

SARS-CoV-2 - severe acute respiratory coronavirus 2

TUG - Timed Up and Go

\section{Authors contributions}

CT performed functional evaluations, contributed to data collection, analysis and interpreted the results. $\mathrm{EV}$ contributed to data collection. VC and MI contributed to interpretation of data and to manuscript supervision. All authors read and approved the final version of the manuscript.

\section{Acknowledgments None.}

Funding None.

\section{Conflict of Interest}

The authors declare to have no conflict of interest.

\section{Ethical Publication Statement}

We confirm that we have read the Journal's position on issues involved in ethical publication and affirm that this report is consistent with those guidelines.

\section{Corresponding Author}

Caterina Tramonti, Fondazione Don Carlo Gnocchi Onlus. Via Fontevivo 126, La Spezia, Italy. Tel.: +39 (0) 187 5451253; Fax: 01875451297. 
ORCID iD: 0000-0002-7560-8964

E-mail address: caterina.tramonti@gmail.com

E-mails and ORCID iD of co-authors

Valentina Carli:vcarli@dongnocchi.it

ORCID iD: 0000-0001-7306-9285

Elena Vatteroni: evatteroni@dongnocchi.it

ORCID iD: 0000-0001-9491-8792

Francesco Iacopini: f iacopini@libero.it

ORCID iD: 0000-0001-5879-8869

Martina Iardella: miardella@dongnocchi.it

ORCID iD: 0000-0003-3927-7018

\section{References}

1. Casadevall A, Pirofski LA. The convalescent sera option for containing COVID-19. J Clin Invest. 2020 Apr 1;130(4):1545-1548. doi: 10.1172/JCI138003.

2. Wang D, Hu B, Hu C, Zhu F, Liu X, Zhang J, Wang B, Xiang H, Cheng Z, Xiong Y, Zhao Y, Li Y, Wang $X$, Peng Z. Clinical Characteristics of 138 Hospitalized Patients With 2019 Novel CoronavirusInfected Pneumonia in Wuhan, China. JAMA. 2020 Mar 17;323(11):1061-1069. doi: 10.1001/jama.2020.1585. Erratum in: JAMA. 2021 Mar 16;325(11):1113.

3. Tsivgoulis G, Palaiodimou L, Katsanos AH, Caso V, Köhrmann M, Molina C, Cordonnier C, Fischer U, Kelly P, Sharma VK, Chan AC, Zand R, Sarraj A, Schellinger PD, Voumvourakis KI, Grigoriadis N, Alexandrov AV, Tsiodras S. Neurological manifestations and implications of COVID-19 pandemic. Ther Adv Neurol Disord. 2020 Jun 9;13:1756286420932036. doi: 10.1177/175628642 0932036.

4. Fernandez CE, Franz CK, Ko JH, Walter JM, Koralnik IJ, Ahlawat S, Deshmukh S. Imaging Review of Peripheral Nerve Injuries in Patients with COVID-19. Radiology. 2021 Mar;298(3):E117E130. doi: 10.1148/radiol.2020203116. Epub 2020 Dec 1.

5. Montalvan V, Lee J, Bueso T, De Toledo J, Rivas K. Neurological manifestations of COVID-19 and other coronavirus infections: A systematic review. Clin Neurol Neurosurg. 2020 Jul;194:105921. doi: 10.1016/j.clineuro.2020.105921. Epub 2020 May 15.

6. Medical Research Council of the United Kingdom. Aiak to Examination of the Peripheral Nervous System: Memorandum No 45. Palo Alto, Calif: Pedragon House; 1978.

7. Fayazi M, Dehkordi SN, Dadgoo M, Salehi M. Testretest reliability of Motricity Index strength assessments for lower extremity in post stroke hemiparesis. Med J Islam Repub Iran. 2012 Feb;26(1):27-30.

8. Schoene D, Wu SM, Mikolaizak AS, Menant JC, Smith ST, Delbaere K, Lord SR. Discriminative ability and predictive validity of the timed up and go test in identifying older people who fall: systematic review and meta-analysis. J Am Geriatr Soc. 2013 Feb;61(2):202-8. doi: 10.1111/jgs.12106. Epub 2013 Jan 25.

9. Collin C, Wade DT, Davies S, Horne V. The Barthel ADL Index: a reliability study. Int Disabil Stud. 1988;10(2):61-3. doi: 10.3109/0963828880916 4103.

10. Algahtani H, Subahi A, Shirah B. Neurological Complications of Middle East Respiratory Syndrome Coronavirus: A Report of Two Cases and Review of the Literature. Case Rep Neurol Med. 2016;2016:3502683. doi: 10.1155/2016/3502683. Epub 2016 Apr 28.

11. Tsai LK, Hsieh ST, Chao CC, Chen YC, Lin YH, Chang SC, Chang YC. Neuromuscular disorders in severe acute respiratory syndrome. Arch Neurol. 2004 Nov;61(11):1669-73. doi: 10.1001/archneur. 61.11.1669.

12. Tsai LK, Hsieh ST, Chang YC. Neurological manifestations in severe acute respiratory syndrome. Acta Neurol Taiwan. 2005 Sep;14(3):113-9..

13. Kim JE, Heo JH, Kim HO, Song SH, Park SS, Park TH, Ahn JY, Kim MK, Choi JP. Neurological Complications during Treatment of Middle East Respiratory Syndrome. J Clin Neurol. 2017 Jul;13(3):227-233. doi: 10.3988/jcn.2017.13.3.227

Submission: June 30, 2021

Revision submitted: August 16, 2021 Accepted for publication: August 16, 2021 